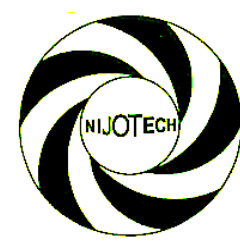

Nigerian Journal of Technology (NIJOTECH)

Vol. 36, No. 1, January 2017, pp. 172 - 177

Copyright@ Faculty of Engineering, University of Nigeria, Nsukka,

Print ISSN: 0331-8443, Electronic ISSN: 2467-8821

www.nijotech.com

http://dx.doi.org/10.4314/njt.v36i1.22

\title{
RECEIVE SIGNAL STRENGTH PREDICTION IN THE GSM BAND USING WAVELET DECOMPOSITION
}

\author{
A. Danladi1 ${ }^{1,}$, N. W. Silika ${ }^{2}$ and P. G. Vasira ${ }^{3}$ \\ 1,2,3 Department of Pure and Applied Physics, Adamawa State University, Mubi , AdAmawa State. NiGERIA \\ E-mail addresses: ${ }^{1}$ deshalangs3g@yahoo.com, 2silikwawaba@yahoo.com,3ask4glandu@gmail.com
}

\begin{abstract}
In this work, GSM receive signal strength was monitored in an indoor environment. Samples of GSM receive signal strength was measured on a Mobile Equipment (ME). One-dimensional multilevel wavelet decomposition technique was used to predict the fading phenomenon of the GSM receive signal strength measured. Wavelet prediction revealed that the GSM receive signal strength is attenuated due to the slow fading phenomenon, which fades about 3 times faster than the radio wavelength. The prediction is further validated using probability density functions in terms of Gaussian and Rayleigh distributions. It is observed that, significant part of the signal strength measured is dominated by good signal ($101 \mathrm{dBm}$ to $-74 \mathrm{dBm}$ ) with an average of $-88.8842 \mathrm{dBm}$ and the signal strength followed more of Gaussian than Rayleigh distribution. This confirmed the wavelet prediction.
\end{abstract}

\section{Keywords: Decomposition, De-Noising, Reconstruction, Fading, Wavelet and Signal Strength}

\section{INTRODUCTION}

A GSM network consists of some functional components, whose function and interface are defined to achieve communication between two subscribers. The GSM components are mainly divided into two that is the Operation Support Subsystem (OSS) and ME. The OSS is further classified into sub-categories such as Base Station Subsystem(BSS) and the Network Switching Subsystem (NSS) and the BSS consists of Home Location Register (HLR), Authentication Center (AUC), Equipment Identification Register (EIR) and Visitors Location Register (VLR). The BSS also consists of Base Station Controller (BSC) and Base Transceiver Station (BTS).In this work, detailed function or operational principles of the GSM architecture is not our focus. However, attention shall be paid to the monitoring and investigation of the signal interface between the BTS and ME, particularly, on signal strength fading because the signal may not be hitched free from transmission impairments. This is important since the fundamental aim of any GSM service provider is to provide outstanding quality of service (QoS) with little or no interruptions. Ideal propagation means equal propagation in all directions with $100 \%$ of QoS. Unfortunately, in real life situation, it is impossible to achieve this due to some certain factors that hamper effectiveness of the propagation between the GSM (BTS) and the ME. This leads Subscriber, to experience call difficulties such as frequent call drop, poor intra and inter connectivity, cross talk in call conversation, especially, in indoor environments. To overcome these problems mentioned above, it is necessary to constantly monitor the GSM signal strength or the QoS, in order to come out with accurate bill of quantities of the activity on our networks, and in addition GSM signal is discrete in nature unlike continuous signal that can easily be monitored. The demand for GSM service is rapidly growing in the entire world. As the number of subscribers of GSM mobile equipment increases, the spectral efficiency becomes so essential. This is because the frequency allocation is a limited resource. Greater capacity or excellent QoS is achieved as a result of less frequency reuse [4]. High spectral efficiency can be realized by reusing frequency in a dense or complex environment such as outdoor or indoor areas; thick forest, high buildings, large water surface, other geographical features and areas consisting of walls, furniture's and floors which may scatter the signal.

This work, propose to use one-dimensional multilevel wavelet technique to predict the GSM receive signal strength attenuation in terms of slow or fast fading in an indoor environment other than the known general techniques such as deterministic, tray tracing and empirical methods which were previously used in most literatures. The following objective shall be realized: to collect samples of GSM receive signal strength in $\mathrm{dBm}$, decompose, de-noise and reconstruct the receive signal strength measured, compare the measured and approximated receive signal strength and finally, identify 
which type of fading phenomenon attenuates the signal strength received in the study area.

In [2], the authors used one dimensional multilevel wavelet to detect the signal attenuation with reference to multipath and shadow fading, but did not group and predict the average power received in their study area. In [3], a simple approach to a statistical path loss model for indoor communications was developed using power law. The work concluded that the model can be used to obtain basic information on how to estimate the power budget of communication system in a given environment. Also, the work extracted some vital information about the signal fading, but did not classify the characteristics of the signal fading in terms of the fast or slow phenomenon. In [5], a research was conducted on measuring and characterizing the indoor channel for IEEE 802.11 for a WLAN at $2.4 \mathrm{GHz}$, the measurement was conducted in close and open corridors. The authors observed that the signal fading is normally distributed at $5 \%$ significant level, while in [6], another work was carried out on the measurement of signal strength to predict the effect of walls, office partition, floors and building layout on a specific frequency $914 \mathrm{MHz}$. The works revealed that floor attenuates the signal strength more than the other factors.

\section{PROPAGATION MODEL}

The model incorporates the following parameters as given in Equation (1)

$$
P_{L}=x=10 \gamma \log \left(\frac{d}{d_{o}}\right)+X_{\sigma}
$$

In (1), $x$ is the unit loss, $\gamma$ is the power delay index, $d$ is the distance between the transmitter and the mobile equipment, $d_{o}$ is the reference distance and $X_{\sigma}$ is the Normal or Gaussian random variables with zero or negative mean. In the case of no fading, the random variables become zero and when the random variables have Gaussian distribution and high value of $\sigma(\mathrm{dB})$, the receive signal strength may result in Log - Normal distribution. If none of these conditions are applied then, receive signal strength is likely to follow fast fading which may come inform of Rayleigh distribution [1, 21 23].

Slow fading phenomenon occurs due to the diffraction and large reflection from buildings, mountains or other factors and it can be evaluated using (2)

$$
X_{\sigma}(x, \sigma)=\frac{1}{\sqrt{2 \pi}} e^{-\frac{(x-\mu)^{2}}{2 \sigma^{2}}}
$$

While, Log - Normal distribution may be expressed as a continuous probability distribution function given by:

$$
N=\frac{1}{x \sigma \sqrt{2 \pi}} e^{-\frac{(\operatorname{In} x-\mu)^{2}}{2 \sigma^{2}}}
$$

Usually, the fast fading phenomenon follows the Rayleigh distribution depending on the factors applicable to the situation. Rayleigh is expressed as given in (4):

$$
\varphi_{o}(x, \sigma)=\frac{x}{\sigma^{2}} e^{-\frac{x^{2}}{2 \sigma^{2}}}
$$

Fast fading phenomenon is characterized by reflection of local objects or movement of ME around these objects which can cause fast fluctuation of the signal amplitude. For example, signal reflected by the large water surface, glasses, or vehicle movement.

\section{STUDY AREA AND METHOD OF DATA COLLECTION}

The data was collected on the ground floor of a three storey building in a room measuring $14 \mathrm{x} 14 \mathrm{fts}$ size. The room contains; furniture's like table, chairs, bed, clothes hanged on the wall, boxes, standing mirror and other materials. The study area is located at Karewa in Yola town, Yola is the State capital of Adamawa State of Nigeria, the city falls within Sudan Savannah belt vegetation zone, the zone is made of Mountains, Streams, dry land interspaced by shrubs and woody plants (Mohogani, Neem, Shear butter, Cashew, Guava, Mango, Tamarin and others) typically, of $7-12 \mathrm{~m}$ height. Yola lies on longitude $9^{\circ} 20^{\prime} \mathrm{N}$ and Latitude $12^{\circ} 30^{\prime} \mathrm{E}$. The study area has several GSM BTSs installed around residential areas; All the BTSs are higher than the roof height. Samples of GSM receive signal strength in $(\mathrm{dBm})$ were collected in 1,030s at an interval of $5 \mathrm{~s}$ each, using NETWORK SIGNAL PRO software, down loaded from ANDROID play store of a ME (Samsung Galaxy Tab 4). The software is available only on Android Smart Phones neither on IOS nor Windows and it has some additional features that provide other important information about the network as summarized in Table 1.

Table 1: Network Signal Info Feature

\begin{tabular}{ll}
\hline Features & \multicolumn{1}{c}{ Information } \\
\hline Network Operator & MTN/NG \\
SIM Operator & 30 (MNC) \\
Network Type & EGDE*220kbps \\
Network Strength & $-87 \mathrm{dBm}$ \\
Data State & Data Connected \\
Data Activity & Upload/Download \\
Cell & 53406 (Cell ID), 14217 \\
MNC & 30 \\
MCC & 621 \\
Country Code & Ng \\
External IP & 197.210 .16 .157 \\
Phone Type & GSM \\
Transmitter & $1033 m$ \\
Distance & \\
MNC (Mobile & MCC (Mobile Country Code) \\
Network Code) & \\
\hline
\end{tabular}

The 'system info' is calibrated from $-113 \mathrm{dBm}$ to $-47 \mathrm{dBm}$ where, from $-113 \mathrm{dBm}$ to $-102 \mathrm{dBm},-101 \mathrm{dBm}$ to $75 \mathrm{dBm}$ and $-74 \mathrm{dBm}$ to $-47 \mathrm{dBm}$ and are classified as 
weak, good and excellent receive signal strength respectively. Moreover, the smaller the number of the $\mathrm{dBm}$ received by the ME, the worse the reception or QoS. Therefore, $-47 \mathrm{dBm}$ is much better than $-113 \mathrm{dBm}$. The samples of the GSM receive signal strength collected is depicted in Fig. 1, presented as signal strength measured $(\mathrm{dBm})$ against time (s). It can be seen that, the signal strength is inconsistent; the variation may be attributed to fading cause due to transmission impairments.

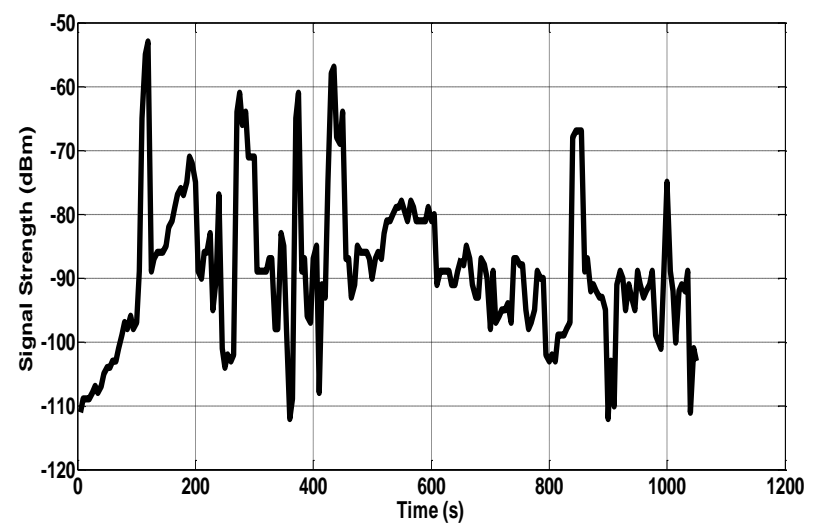

Fig. 1: Samples of Signal Strength

\section{WAVELET DECOMPOSITION AND PREDICTION ALGORITHM}

Wavelet simply means wave with short duration with an average value zero. Wavelet tool is one of the vital tools used in signal processing which can analyze smallest part of the signal that older tools like Fourier transform could not be able to analyze. Generally, Wavelet tool is used in many varieties of works but in this work, the tool is used as a filter. There are different types of Wavelet tools; this includes Debauches, Haar, Morley and Bio and many others. To successfully achieve the filtering process a couple of things have to be done. For example, decomposition and reconstruction of the discrete GSM signal received.

\subsection{Discrete Signal Decomposition and Reconstruction Process}

In order to achieve signal full decomposition process, the discrete signal strength received has to be decomposed into two parts called approximated and the detail coefficient. The decomposed signal has to be passed through the quadrature mirror filters which consist of high and low pass filters given by the symbols $g(n)$ and $\mathrm{h}(\mathrm{n})$ respectively. Usually, the approximated signal passes through the low-pass filter and the detail signal passes through high-pass filter as depicted in Fig. 2, assuming the GSM signal strength to be decomposed is given by $\mathrm{y}[\mathrm{n}]$. The approximated signal is denoted as $\mathrm{cA}_{J}$ and the detailed is denoted by $\mathrm{cD}_{j}$ both on octave respectively.

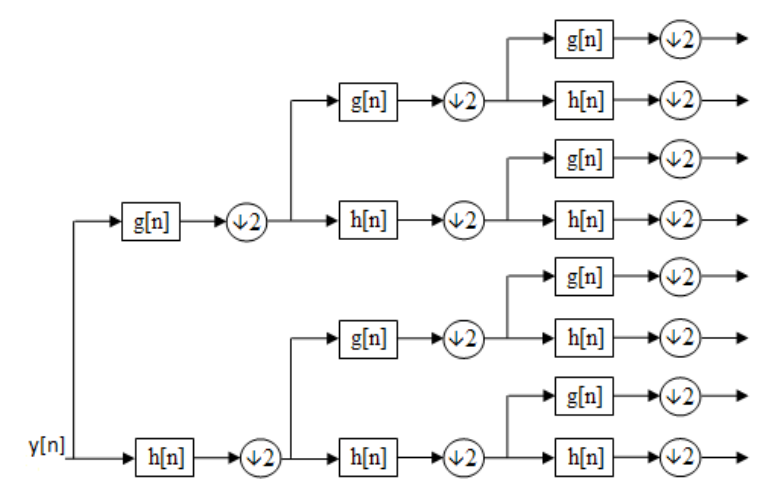

Fig. 2: Wavelet Decomposition Levels

Reconstruction of the decomposed signal can be achieved by assembling back the components of the signal into original signal without losing meaningful information sometimes referred to as inverse discrete wavelet transform (IDWT). However, during filtering, attention must be paid to the choice of the appropriate filters so that, the signal structure will not be compromised. Because, at that time the information about the signal may not be clear.

Full wavelet decomposition of the attenuated signal measured provides information about the time and the frequency of the signal at numerous scales. Thus, the signal can be seen in detailed and approximated form both on the octave axis $(\mathrm{j}, \mathrm{J})$ respectively as shown in Fig. 3. The approximated signal is situated on the upper scale ranging from $1 \leq \mathrm{J} \leq \mathrm{Jn}$, with low frequencies considered to be the vital part of the signal and the detailed on the lower scale ranging from $1 \leq \mathrm{j} \leq \mathrm{Jn}$. with high frequencies considered to be the unwanted part of the signal (noise). The wavelet coefficients $A_{x}(J, K), D_{x}(J, k)$, are derived from the relation below after full decomposition.

$$
\psi_{a b}(t)=\frac{1}{\sqrt{a}} \psi\left(\frac{t-b}{a}\right)
$$

In (5), $a$ is the positive number which defines the scale and $b$ is the real number that defines the shift [24]. Equation (5) sometimes called child wavelet derived from the mother wavelet. But full wavelet decomposition (FWD) is composed of the combine wavelet coefficients of the approximated and the detailed signal as given in (6)

$$
y(n)=A_{x}(J, k) \psi_{o}(J, k)+\sum_{k-1}^{j} D_{x}(j, k) \varphi_{o}(j, k)
$$

In (6), $\mathrm{y}$ is decomposed using Haar at level 3, this means that, $\mathrm{n}=3$. At this level the decomposition produced $2^{n}=2^{3}=8$ coefficients. Haar is chosen for this suppose because of the following reasons; physical appearance of the experimental data and the wavelet Haar, coefficient of correlation is approximately equal and the wave's energy is almost the same. The following gives numerical 
example of the GSM signal strength decomposed and reconstructed. Normally, Haar Wavelet has the following transforms at level- 1 as

i. Running average; that is the average sum of the two successive signal received by the ME.

$$
C_{m}=\frac{P_{2 m-1}+P_{2 m}}{\sqrt{2}}
$$

ii. Running difference; that is the average difference of the two successive signal received by the $\mathrm{ME}$

$$
d_{m}=\frac{P_{2 m-1}+P_{2 m}}{\sqrt{2}}
$$

Where $m=1,2,3,4, \ldots . n / 2$

For example, consider the first 12 successive receive signal strength received by the $\mathrm{ME}$ as $\mathrm{P}_{1}, \mathrm{P}_{2}, \mathrm{P}_{3}, \mathrm{P}_{4}, \ldots$. $\mathrm{P}_{12}$ which corresponds to the $-111 \mathrm{dBm},-109 \mathrm{dBm}$, $109 \mathrm{dBm},-109 \mathrm{dBm},-108 \mathrm{dBm},-107 \mathrm{dBm},-108 \mathrm{dBm},-$ $107 \mathrm{dBm},-105 \mathrm{dBm},-104 \mathrm{dBm},-104 \mathrm{dBm}$ and $-103 \mathrm{dBm}$.

To decompose and reconstruct the signal, there is a need to satisfy the conditions in (i) and (ii) above using (9), (10) and (11).

$$
\begin{array}{r}
C_{m}^{2}: C_{1}=\sqrt{2}\left(\frac{P_{1}+P_{2}}{2}\right), C_{2}=\sqrt{2}\left(\frac{P_{3}+P_{4}}{2}\right), \\
C_{3}=\sqrt{2}\left(\frac{P_{5}+P_{6}}{2}\right), \ldots, C_{6}=\sqrt{2}\left(\frac{P_{11}+P_{12}}{2}\right)
\end{array}
$$

The introduction of $\sqrt{2}$ is to ensure sufficient energy conservation.

$$
\begin{aligned}
& C_{m}^{2} .110 \sqrt{2} \mathrm{dBm},-109 \sqrt{2} \mathrm{dBm},-108.5 \sqrt{2} \mathrm{dBm}, \\
& -108.5 \sqrt{2} \mathrm{dBm},-104.5 \sqrt{2} \mathrm{dBm},-103.5 \sqrt{2} \mathrm{dBm} \\
& d_{m}^{2}: C_{1}=\sqrt{2}\left(\frac{P_{1}+P_{2}}{2}\right), d=\sqrt{2}\left(\frac{P_{3}+P_{4}}{2}\right), \\
& d_{3}=\sqrt{2}\left(\frac{P_{5}+P_{6}}{2}\right), \ldots, d=\sqrt{2}\left(\frac{P_{11}+P_{12}}{2}\right) \\
& d_{m}^{2} . . \sqrt{2} \mathrm{dBm}, 0 \mathrm{dBm}, 0.5 \sqrt{2} \mathrm{dBm},-0 . .5 \sqrt{2} \mathrm{dBm} \\
& -0.5 \sqrt{2} \mathrm{dBm},-0.5 \sqrt{2} \mathrm{dBm} \\
& P=C_{m}^{2}-d_{m}^{2} \\
& \left(\begin{array}{c}
-111,-109,-109,-109,-108,-107,-108,-107,-105, \\
-104,-104,-103
\end{array}\right) \mathrm{dBm} \\
& =110 \sqrt{2},-109 \sqrt{2},-108.5 \sqrt{2},-108.5 \sqrt{2},-104.5 \sqrt{2},-103.5 \sqrt{2} \\
& -1,0,0.5,0.5,0.5,0.5,0.5 \mathrm{dBm} \\
& P=\frac{C_{1}+d_{1}}{\sqrt{2}}, \frac{C_{1}-d_{1}}{\sqrt{2}}, \ldots \ldots, \frac{C_{n / 2}+d_{n / 2}}{\sqrt{2}}, \frac{C_{n / 2}-d_{n / 2}}{\sqrt{2}} \\
& P=\frac{110 \sqrt{2}+\sqrt{2}}{\sqrt{2}}, \frac{110 \sqrt{2}-\sqrt{2}}{\sqrt{2}}, \ldots \ldots, \frac{103.5 \sqrt{2}+0.5 \sqrt{2}}{\sqrt{2}}, \\
& \frac{103.5 \sqrt{2}-0.5 \sqrt{2}}{\sqrt{2}}
\end{aligned}
$$

Where $P_{1}=-111 \mathrm{dBm}, \mathrm{P}_{2}=-109 \mathrm{dBm}$. . . . . $\mathrm{P}_{11}=$ $104 \mathrm{dBm}, \mathrm{P}_{12}=-103 \mathrm{dBm}$

As it can be seen, $\mathrm{P}$ is successfully reconstructed without compromising the signal structure. The same principles can be applied to the other parts of the decomposition levels.

\subsection{Prediction process}

Several prediction methods such as deterministic, ray tracing and empirical techniques [4, 7 - 17], have been developed to predict path loss in open or indoor area. In most literatures, these methods are adopted for path loss prediction but in this work, an alternative method of prediction is employed called wavelet prediction technique. The prediction is done by filtering the unwanted part of the signal received. The ideal wavelength $(\lambda)$ of propagation of the study area may be obtained as $\lambda=C / f$; where $\mathrm{C}$ is the speed of the propagated wave and it can be evaluated by $C=d / t$; where, $d$ is the distance between the BSS and the ME given by $d=\left(x_{B S}^{2}+x_{M E}^{2}\right)^{1 / 2}$ [18-19]. Having obtained the total amount of the signal strength received in the study area from expression (13).

$$
\sum_{i=1}^{n} P_{i}(d B m)=P_{1}+P_{2} \ldots+P_{n}
$$

Comparing the received and the approximated $\left(\mathrm{P}_{\mathrm{a}}\right)$ signal strength, the ratio $\mathrm{P}_{\mathrm{i}} / \mathrm{P}_{\mathrm{a}}$ is equal to $3: 1$, this means that, the frequency of the signal strength received fades 3 times and the wavelength becomes $\lambda_{n}=C a / f$ greater than the normal wavelength of the propagation. Where $\alpha$, is the fading factor and $\lambda_{\mathrm{n}}$ is the faded wavelength. Therefore, it can be deduced that, the ratio of the signal strength approximated at level 3 fades about 3 times the wavelength of the propagation. It is worthy to say that the signal received corresponds to the slow fading scale as shown in Fig. 3.

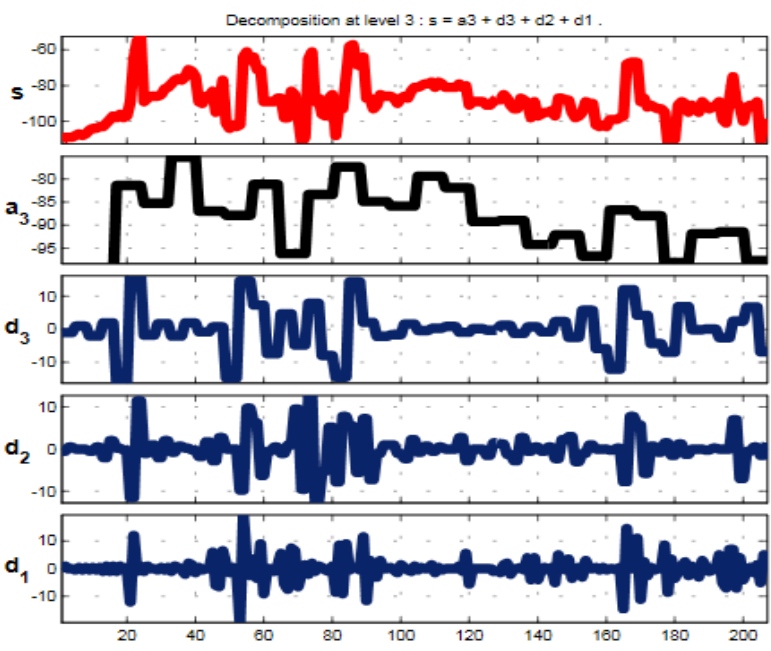

Fig. 3. Wavelet Prediction of the Signal Strength Received (s) Showing Detailed $\left(d_{1}, d_{2}, d_{3}\right.$ ) and Approximated ( $\left.a_{3}\right)$ Signal

Therefore, to determine the propagation error, the root mean square error between the approximated and the received signal, is obtained using expression (14) as $2.884 \mathrm{~dB}$ 


$$
R M S E=\sum_{i}^{n}\left(\sqrt{\frac{\left(P-P_{a}\right)^{2}}{n}}\right)
$$

In (14), $n$ and $P_{a}$ is the number of the empirical data and the approximated signal respectively.

As described in the model Equation (1), we further went ahead to validate our wavelet prediction using probability distribution function (PDF) of the signal strength received in terms of Rayleigh and Gaussian distribution as shown in Fig. 4. The signal strength follows more of Gaussian distribution than Rayleigh distribution. This is obtained by comparing the mean of signal strength measured and the mean of the PDFs as given in Table 2.

Table 2: Comparison of the Mean of receive signal strength and mean of the distributions

\begin{tabular}{cc}
\hline Items & Mean $(\mathrm{dBm})$ \\
\hline Signal Strength & -88.8842 \\
Gaussian Distribution & -88.8842 \\
Rayleigh Distribution & -80.1266 \\
\hline
\end{tabular}

The mean of the signal strength is obtained using (15):

$$
\text { Mean }=\frac{\sum f x}{\sum f}
$$

Where $\mathrm{x}$, presents the signal strength measured and $\mathrm{f}$, is the total number of the signal strength measured.

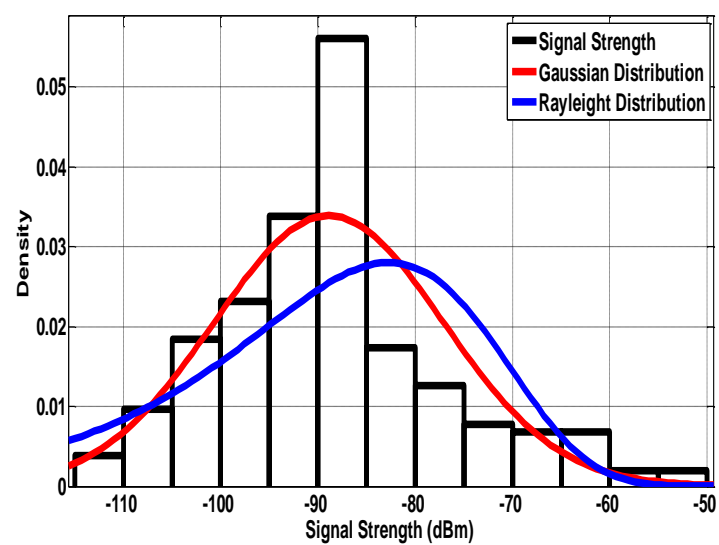

Fig. 4 Probability Density Functions of the Signal Strength Received

Yarkony and Blaustein [1] suggested a model using slow and fast fading and also, takes into account inner objects in the study area but arrived at average prediction error of $7.2 \mathrm{~dB}$ and [20] did not consider types of fading phenomenon but arrived at an error of $8.38 \mathrm{~dB}$, both errors are obtained at the first floor of a storey building. In comparison to what is obtained in this work, wavelet decomposition technique has more efficient prediction performance better than what is reported in $[1,20]$ by $4.316 \mathrm{~dB}(59.1 \%)$ and $5.496 \mathrm{~dB}(65.6 \%)$ respectively.

\section{CONCLUSION}

In this work, one-dimensional multilevel wavelet was used to predict the GSM signal strength attenuation in an indoor environment in terms of fading phenomenon, it is obtained that, the GSM receive signal strength is attenuated by the slow fading phenomenon since signal strength followed more of Gaussian than Rayleigh distribution and the average signal strength received is 88. $8842 \mathrm{dBm}$,this means, significant part of the signal strength received in the study area is a good signal.

\section{REFERENCES}

[1] N. Yarkony and N. Blaunstein, Prediction of Propagation Characteristics in Indoor Radio Communication Environments, Progress in Electromagnetic Research, PIER 59, 151 - 174, 2006

[2] Biplab Sikdarand Xiaobo Long. Wavelet Based Detection of Shadow Fading in Wireless Networks. Global Telecommunication Conference, 26 - 30 November, 2007

[3] Constantino Perez-vega and Jose Luis Garcia. "A simple approach to a statistical path loss model for Indoor". Communication Engineering Department University of Cantabria Av. De los castros s/u. 39005. Santander, Spain. Pp 617 - 623

[4] Yu - Huei, T. Wen - Shyang, H. and Ce - Kuen, S. The influence of propagation in a live GSM network, Journal of electrical eng.Vol 7 No.1, 1 - 7. 2009.

[5] Robert Akl, Dinesh Tummala and Xinrong Li. Indoor Propagation Modeling at 2.4GHz for IEEE 802.11 Network. Sixth lasted international multiconference on wireless and optical comm. Wireless network and imagining technologies. Banff $\mathrm{AB}$. Canada 3-5, July, 2006.

[6] Scott, Y. Seidel and Theodore, S. Rapapport. 914MHz path Loss Prediction Models for Indoor Wireless Communication in Multi Floored Building. IEEE Transaction on antenna and propagation. February, 1992.

[7] Sami, A. M. Path Loss Propagation Model Prediction for GSM network planning. International Journal of Computer Applications, Vol. 84, No.7, 2013.

[8] European Cooperation in the Field of Scientific and Technical Research Euro- Cost 231. Urban Transmission Loss Model for Mobile Radio in the 900 and 1800MHz Bands. "Revision 2" COST 231 TD (90)119 Rev. 2, , The Hague, the Netherlands, September, 1991.

[9] Goldsmith, A. "Wireless Communication", USA, Cambridge University Press2005

[10] Wu, J., and Yuan, D. Yuan. Propagation Measurements and Modeling in Jinan City. IEEE International Symposium on Personal, Indoor and Mobile Radio Communications, Boston, MA, USA, 
Vol. 3, 1157- 1159, 8-11 September, 1998.

[11] Nadir, Z. I. Aeng, N. F., and Touati F. Path Loss Determination Using Okumura-Hata Model and Spline Interpolation for Missing Data for Oman. Proceedings of the World Congress on Engineering, Vol. 1, 2-4, 2008.

[12] Hata, M. Empirical Formula for Propagation Loss in Land Mobile Radio Services. IEEE Transaction on Vehicular Technology Vol. 29, No. 3, 1980.

[13] Okumura Y. Field Strength and Its Variability in VHF and UHF Land-Mobile Radio Service. Review of the Electrical Communications Laboratory, Vol.16, 9-10, 1968.

[14] Erceg, V., Greenstein, L. An Empirical Based Path Loss Model for Wireless Channels in Suburban Environments. IEEE Journal on Selected Areas of Communication. Vol. 17, 1205-1211, 1999.

[15] Medeisis A. and Kajackas A. On the Use of the Universal Okumura-Hata Propagation Predication Model in Rural Areas. Vehicular Technology Conference Proceedings, VTC Tokyo, .Vol.. 3, 18151818, 2000.

[16] Xiaobo L. and Biplab S.. "Wavelet Based Detection of Shadow Fading in Wireless Networks". Electrical, Computer and System Engineering Rensselaer Polytechnic Institute, 110 8th Street, Troy NY 12180.

[17] Yuvraj S. Comparison of Okumura, Hata and COST231 on the bases of the path loss and signal strength. International Journal of Computer and
Application, Vol. 59, 11, 2012.

[18] Danladi A. Wavelet based path loss modeling for global system for mobile communication in an urban environment. International Journal of Science and Research Vol. 3 No. 7, 1929 - 1932, 2014.

[19] Danladi A. and Natalia V. A. Measurement and Modeling of Path Loss for GSM Signal in a Sub Urban Environment over Irregular Terrain-Review. International Journal of Science and ResearchVol.3, No. 8, $611-614,2014$.

[20] Molley A. J. and Keenan J. M. P., Personal Communication Radio Coverage in Building at $900 \mathrm{MHz}$ and $1700 \mathrm{MHz}$, IEEE Electronics Letters, Vol. 24, No. 12, 763 - 774, 1999.

[21] Bertoni H.L., Radio Propagation for Modern Wireless System, Prentice Hall PTR, New January, 2013

[22] Samir M. Hameed, Indoor Propagation Modeling for Wireless Local Area Network (WLAN), Engineering Conference of Control, Computer and Mechatronics, Vol. 11, No. 1, 97 - 105, 30 -31 Jan, 2011

[23] Jadhavar B. R. and Sontakke T. R., $2.4 \mathrm{GHz}$ Propagation Models for Indoor Wireless Communications within Building, International Journal of Soft Computing and Engineering, Vol. 2, Issue 3, 108 - 113, July, 2012.

[24] Daniel T. I. Lee and Akio Yamamoto, Wavelet Analysis: Theory and Applications, Hewlett-Packard Journal, 1994. 